\title{
The Power of Consumer Activism and the Value of Immunization Registries in a Pandemic
}

\section{Michael Popovich, Todd Watkins, Ousswa Kudia}

\author{
Scientific Technologies Corportation, Phoenix, Arizona, United States
}

\section{Objective}

If public health agencies used direct communication channels to individuals by building on existing immunization networks, the public would receive correct information quicker during a pandemic. Furthermore, there is value that can be leveraged from social networks to advance public health efforts to manage disease events and encourage consumers being more proactive in managing their own health care.

\section{Introduction}

Epidemiologists and public health professionals work to ensure the risk and impact of existing and emerging diseases are minimized and do not turn into a pandemic. Timely and accurate information has become imperative as the world has become more interconnected through travel and technology.

Recently, new information systems have played a key role in improving public health's early warning and case management for disease outbreaks. Improved analytics to predict risk in populations have helped researchers open new doors to disease cures and medicines. The role of technology and public health to support these efforts has become more valuable.

Health information systems are traditionally used for electronic medical records or payer billing systems and are not paired with technology advancements. Efforts today to link information and technology to engage consumers are championed by health plans and healthcare providers. Empowering individuals to be proactive when presented with their medical records is not a simple problem to solve. Information must be actionable but it still may not achieve the desired success.

What if the health community engaged consumers with a social mission to help them fight disease outbreaks by becoming frontline activists to report occurrences and outcomes, and become "intelligent connections" to extend the right information to their social networks? This would encourage consumer technology to be better integrated with health information technology with continued investment in sustaining critical public health ecosystems.

A large part of health information systems are immunization information systems (IIS) where administered vaccines are documented in a confidential computer based system in a specific geographic area [1]. The IIS can be used for disease surveillance purposes and provide valuable information to public health authorities [2]. Recently, MyIR was created where any IIS, pharmacy or provider can provide patients direct access to family immunization records. Providers can communicate to patients using My IR to increase engagement and send vaccine reminders.

\section{Methods}

A public-health engagement approach to empower consumers begins by offering individuals a mission they care about that will contribute to the social good and make them more attentive to their own healthcare. Our approach was to place a mission for every cell phone owner by using a commonly understood health event.

The most significant public health event in the 20th century was the power of vaccines and the most significant action an individual can take to reduce their risk of a vaccine-preventable disease is to stay up-to-date on their immunizations [3]. However, there is a gap between believing in the value of immunizations and ensuring one's immunizations are current. The challenge is to engage individuals, empower them to be advocates of their own health and in an outbreak, become sources of trusted public health messages as they communicate in their social network.

A few experiments were conducted using MyIR.

ISDS Annual Conference Proceedings 2019. This is an Open Access article distributed under the terms of the Creative Commons AttributionNoncommercial 4.0 Unported License (http://creativecommons.org/licenses/by-nc/3.0/), permitting all non-commercial use, distribution, and reproduction in any medium, provided the original work is properly cited. 


\section{Increase Sustainment}

Users who had not used MyIR in over 30 days were contacted. The baseline looked at users that were logging into MyIR more than once a month. The target was non-engaged users which were about 8,000 accounts.

\section{Customer Engagement I}

In November 2017, an email was sent to 7,772 users that asked them, "Did you get your flu shot?" If they clicked Yes, they received positive affirmation. If they clicked No, the message was an encouragement to get their flu shot before Thanksgiving 2017.

\section{Customer Engagement II}

In January 2017, a Healthy Lifestyle page was created within MyIR. It featured a food blogger who offers readers nutritious easy meal ideas.

\section{Outreach Efforts}

MyIR Louisiana users were targeted who had failed to complete the two step enrollment process to access their immunization histories.

\section{Efficacy of Flu}

In April 2018, 212 MyIR users in Washington and Louisiana were asked: Did you get a flu shot this year? Do you feel like you got the flu this year?

\section{Results}

\section{Increase Sustainment}

$27 \%$ of people opened the email and 3.6\% of these individuals used MyIR within 30 days to access their records.

\section{Customer Engagement I}

$9 \%$ answered the question with $80 \%$ saying "YES" they got their flu shot. As a result, 25 new immunizations were administered to these individuals within 60 days.

\section{Customer Engagement II}

A $7.1 \%$ increase in returning users were tracked and a $9.1 \%$ increase in engaged users. There was a $6.7 \%$ increase in average session duration.

\section{Outreach Effort}

556 emails were sent which contained instructions to finalize enrollment for MyIR. There was a 30\% open rate and 50 individuals completed the process.

\section{Efficacy of Flu}

$78 \%$ responded they did receive the flu shot this year. Of these, $61.5 \%$ felt they got the flu this year which equates to a $38.5 \%$ efficacy rate. In February, CDC had determined the interim estimates for the effectiveness of the influenza were 36\% [4].

\section{Conclusions}

Our aim was to show examples where public health agencies using direct communication channels to individuals could increase the efficacy of reaching the public with correct information. It was not designed to prove to be statistically effective but to show the potential of engaging individuals that have access to their immunization records. These early experiments and the growing data 
ISDS 2019 Conference Abstracts

assets in IIS's help create a framework and technical platform to accelerate the potential value of engaging individuals in response plans for pandemic preparedness.

Immunization information systems and technology have reached a point where information is available across wide networks of stakeholders. While health plans, providers and pharmacists struggle to engage their networks, by encouraging patients to be proactive in their healthcare, public health immunization assets may be the tipping point to accelerate this movement.

Continued investment of immunization programs, private sector innovation, and consumer empowerment are essential to evolve and sustain data assets. As these assets create added value to each stakeholder, the investment will create a positive return. The value of this virtual ecosystem is untapped and opportunities to use it to drive down healthcare costs and improve patient outcomes are unlimited.

\section{Acknowledgement}

We would like to thank Theresa Munanga for her editorial assistance and all STC employees, especially those who assisted with the MyIR studies.

\section{References}

1. Centers for Disease Control Prevention (CDC). About Immunization Information Systems. Atlanta: CDC; 2012 May 15 [Cited 06 Jun 2018]. Available from: http://www.cdc.gov/vaccines/programs/iis/about.html

2. Derrough T, Olsson K, Gianfredi V, et al. 2017. Immunisation Information Systems - useful tools for monitoring vaccination programmes in EU/EEA countries, 2016. Euro Surveill. 22(17), 30519. PubMed https://doi.org/10.2807/1560-7917.ES.2017.22.17.30519

3. Centers for Disease Control and Prevention (CDC). 1999. Ten great public health achievements--United States, 1900-1999. MMWR Morb Mortal Wkly Rep. 48(12), 241-43.

https://www.cdc.gov/mmwr/preview/mmwrhtml/00056796.htm. PubMed

4. Flannery B, Chung JR, Belongia EA, et al. 2018. Interim Estimates of 2017-18 Seasonal Influenza Vaccine Effectiveness - United States. February. MMWR Morb Mortal Wkly Rep. 67(6), 180-85.

https://www.cdc.gov/mmwr/volumes/67/wr/mm6706a2.htm. PubMed https://doi.org/10.15585/mmwr.mm6706a2 